\title{
The quality of the tear film in glacuoma patients after phacoemulsification surgery
}

Authors: Sandra Lukić ${ }^{1}$, Dubravka Biuk ${ }^{2,3}$ (mentor)

${ }^{1}$ Faculty of Medicine, Josip Juraj Strossmayer University of Osijek, Osijek, Croatia

${ }^{2}$ Department of Ophthalmology, Osijek University Hospital Centre, Osijek, Croatia

${ }^{3}$ Department of Ophthalmology, Faculty of Medicine, Josip Juraj Strossmayer University of Osijek, Osijek, Croatia

DOI: https://doi.org/10.26800/LV-142-supp5-36

\section{Introduction:}

Glaucoma is a chronic disease that requires permanent topical drug therapy. The negative effects of eye drops on the ocular surface may be due to the action of the active ingredient or preservative. The most commonly used preservative is benzalkonium chloride which is a detergent and then polyquad which is its derivative.

Aim:

The aim of this study is to compare the quality of the tear film in three groups of patients, two of whom use eye drops in therapy that contain the preservatives benzalkonium chloride and polyquad and one that uses preservative-free drops in their therapy.

\section{Materials and methods:}

The study included 60 subjects of both sexes operated on due to cataract by phacoemulsification. To assess tear film quality, each patient underwent a Schirmer test 1, a fluorescein test, and a tear-breakup-time test (TBUT) on the day of the surgery, on the $1^{\text {st }}$ and $7^{\text {th }}$ day after the surgery and one month after the surgery. The difference in the measured tear film before and after phacoemulsification was examined.

\section{Results:}

After phacoemulsification, there is a significant deterioration in the quality of the tear film. The use of benzalkonium chloride in the therapy statistically $(P<0.001)$ reduces the quality of the tear film compared to the therapy in which the preservative polyquad was used and the therapy without preservatives.

\section{Conclusion:}

After cataract surgery by phacoemulsification, the quality of the ocular surface was reduced in all three groups of subjects. In the group of subjects who have the preservative BAK in therapy, the lowest quality of the ocular surface was observed. The best quality of the ocular surface was observed in the group of subjects who do not have a preservative in therapy.

Keywords: benzalkonium chloride, phacoemulsification, tear film quality 